\title{
METALLIC LITHRM BY QUANTUM MONTE CARLO
}

\author{
G.Sugiyama, G.Zeraht, B.J.Alder \\ Lawrence Livermore National Laboratory \\ Livermore, Ca. 94550
}

Litbium was chosen as the simplest known metal for the first application of quantum Monte Carlo methods in order to evaluate the accuracy of conventional one-electron band theories. Lithium bas been extensively studied using such techniques. The KIRR method [1], the linear muffin tin orbital method (LMTO) [2], the augmented spherical wave method (ASW) [3] and a linear combinations of gaussian type orbitals (LCGTO) method [4] agree in their predictions of the equation of state. Thesz results are also consistent with experimental data available at low compressions [5] [8] and agree with quanium-statistica:-models [7] [8] at high pressures.

Band theory calculations have certain limitations in general and specifically in their application to lithium. Results depend on sucb faciors as charge shape approximations (muffin tins), pseudopotentials (a special problem for lithium where the lack of $p$ core states requires a strong pseudopotential), and the forth and parameters chosen for the exchange potential. The galculations are all oneelectron methods in which the correlation effects are included in an ad hoc manner. This approximation may be particularly poor in the high compression regime, where the core states become delocalized. Furthermore, band theory provides only self-consistent results rather than strict limits on thei energies. The quantum Monte Carlo method is a totally different technique using a many-body rather than a mean field approach which yields an upper bound on the energies.

\section{QUANTUM MANY-BODY ALGORITHM}

The Schrỏdinger equation was solved for a system of $M$ fixed lithium atoms and $N=3 M$ electrons using the quantum Monte Carlo algorithm previously developed for the electron gas [9] [10]. This technique does not approximate the $3 N$ dimensional problem by reducing it to a set of equations of lower dimensionality, but solves it exactly within statistical error bars. The algorithm

† permanent address: Commissariat l'energie stomique, Centre d'etudes de LimeilValenton, France

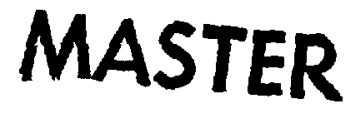


involves seyeral phases of progressively greater accuracy - the variational, the diffusion and the Green's function Monte Carlo methods, which yield upper bounds to the ground state energy, and the released node Green's function Monte Carlo method, which provides exact answers within statistics. In this preliminary study, various trial wavefunctions were explored in order to determine the optimum form for a fixed amount of computational time using the variational and fixed-node schemes. The choice of the trial wavefuncion is a trade off between a simple analytic form which is computationally fast and a more accurate but complex form which is difficuit to sample eficienuly.

Variational Monte-Carlo [11]. Variationally, the total energy of a system of Hamiltonian $\mathbf{B}$ is given by the minimum with respect to the set of all possible trial functions $\boldsymbol{\Psi}_{\boldsymbol{T}}$ of

$$
E=\operatorname{Mia}_{T} \frac{\int \Psi_{T} H \Psi_{T}}{\int\left|\Psi_{T}\right|^{2}} .
$$

In practice, $\Psi_{\boldsymbol{T}}$ is a parameterized expressicn for which the integral (1) is computed using the Metropolis Mnnte Carlo algorithm. The resu: is an upper bound on the energy that is dependent upon the nature of the trial wave function. The standard form of $\Psi_{T}$ consists of a Slater determinant of one-body states multiplied by a pair product Jastrow factor which incorporates two-body correlation effects. For litiium, three differeat forms were used for the elen: nnts of tne determinant. In the simpler cases, the localized states were taken to $\mathrm{t}$-Gaussians with a width parameter and the delocalized states were treated as pla :e waves. In the third case, a more complex form was generated from band theory harge densities as discussed below.

Diffusion Monte Carlo. The diffusion Monte Carlo algorithm [12] computes a more accurate solution of the Schroddinger equation using a trial function generated by the variational Monte Carlo technique. The Schrödinger equation in imaginary time is treated as a difusion equation with the potential acting as a branching birth and death process. The solution converges exponentially to the grolind state. The wavefunction $\Psi$ satisfies

$$
\text { H } \Psi(\vec{R}, t)=\left\{-\sum_{i=1}^{N} \frac{A^{2}}{2 m} \nabla_{i}^{2}+V(\vec{R})-E_{T} \mid \Psi(\vec{R}, t],\right.
$$

where $\vec{R}$ is the $3 N$ dimensional vector of the electronic coordinates, t is the imaginary time and

$$
V(\bar{R})=\sum_{i<j}^{N} \frac{e^{2}}{r_{i j}}-\sum_{i, \alpha}^{N, N} \frac{Z_{\alpha} e^{2}}{r_{i \alpha}}+\sum_{\delta<\delta}^{N} \frac{Z_{\alpha} Z_{\beta} e^{2}}{r_{\alpha \beta}},
$$

is the potential energy of the solid using standard Coulomb interactions. $E_{T}$ is a constant trial energy which is subtiacted from the potential energy for computational convenience. The sums run over the electronic coordinates $i, j$ and the fixed atomic lattice sites $a, \beta$ with $r_{k}=\left|r_{0}-r_{b}\right|$. A finite simulation cell with periodic boundary conditions is used, with the potential energies evaluated by Ewald summation. 
The wavefunction $\Psi$ can be interpreted as the density of diffusing particies as long as it is everywhere of one sign. This is not the the case for fermion statistics where the many-body wavefunction changes sign at the nodes. However, this difficulty can be overcome by using a trial wavefuncticn $\Psi_{\tau}$, whose nodes act as absorbing barriers to the diffusion process. The probablity density defined by

$$
f(\vec{R}, t)=\psi_{T}(\vec{R}) \psi(\vec{R}, t) \exp \left\{-\left\{E_{T}-E_{0}\right) t\right\} \text {. }
$$

obeys the diffusion equation

$$
\left.h \frac{\partial f(R, 1)}{\partial t}=-\left.\sum_{j=1}^{N} \frac{h^{2}}{2 m} \nabla_{j}\left|\nabla_{j} f-f \nabla_{j} \ln \right| \Psi_{T}\right|^{2}\right)-\left\{\frac{H \Psi_{T}}{\Psi_{T}} E_{T}\right\}
$$

which is derived from the Schrobdinger equation. $E_{0}$ is the exact ground state eigenvalue corresponding to the eigenfunction $\Psi$. This equation is solved in each of the regions bounded by the trial function nodes as before, however now the trial function plays an important role in reducing the brasching term - a proccss known as importance sampling.

The fixed-node approximation imposes the constrairt that the wavefunction has the approximate nodal surface of $\Psi_{\tau}$ leading to a upper bound criterion on the energy. In principle, the nodal surfaces could be varied to obtain the best upper bound on the energy. In general, however, it is difficult to porameterize $\nabla_{T}$ in a systematic fashion. For the electron gas, the dependency upon the location of the nodes of the trial wavefunction was weak [10], however for an accurate upper bound $\Psi_{T}$ should be chosen as close to the true ground state wavefuncticn as is feasible.

Green's Function Monte Carlo. The Schrödinger equation recast into integral form can be solved by Monte Carlo sampling of the exact Green's iunction $G$ [13|. This avoids the error incurred in the diffusion Monte Carlo algorithm by the use of a sbort time step expansion approximatica of $G$. Exact evaluation of $G$ coupled with nodal release leads to the stochastically exact solution of the Schrödinger equation.

Trial wavefunction. The general form for the wavefunction that has been used successfully in previous studies consiz:s of the product of a Slater determinant of single particle orbitals multipled by a Jastrow factor $\mathrm{J}$ :

$$
\Psi_{t}=\operatorname{det}\left|\Phi_{i j}\right| \operatorname{det}\left|\Phi_{i j}\right| \mathbf{J},
$$

where the $\Phi_{i j}$ are the one particle wavefunctions of the Slater determinant, with the superscripts * and - denoting the two possible spin states. The determinantal form provides the required fermion antisymmetry. The Jastrow factor

$$
J=\exp \left(-\sum_{i, j}^{N} y_{i j}-\sum_{i, a}^{N, M_{i}} u_{i a}\right)
$$

involves a sum of the electron-nuclear and the electron-electron pair correlation factors. These exactly incorporate the cusp conditions - the singularities of the wavefunction for zero pair separation due to the coulombic divergence of the potential. The Jastrow factor is computed using the random-phase approximation [11].

\section{DISCLAIMER}

\footnotetext{
This tepont was prepared as an account of work sponsored by an agency of the United States Government. Neither the United States Government nor any agency thereof, nor any of their employees, makes any warranty, expsess or implied, or assumes any lezal liability or responsibitity $f$ or the accuracy, completeness, of usefulness of any information, apparatus, product, or process disclosed, or tepresents that its use would not infringe privately owned rights. Reference herein to any specific commercial product, process, ar service by trade name, irademark, martufacturet, of otherwise does not recessarily constifute of imply its endorsemem, iecurn. mendation, of favoring by the United States Government of any agency thereof. The views and opinions of authors expressed herein do not necessarily state or rellect those of the United States Government or any agency thereof.
} 
Two forms of Slater determinant trial function were implemented. In the first calculation, $\Psi$ consisted of Gaussians with parameterized widths centered about the lattices sites for localized states and plane waves for the delocalized orbitals. At extreme compressions where the 1s state was expected to be delncalized, all the Slater determinant states were taken to be plane waves - a form which should yield a lower energy. Such simple analytical forms have been successfully applied to molecules [12] the electron gas [10] and molecular and metallic hydrogen (with emphasis on the metallic transition) [14]. For highly compressed lithium, however, it was desirable to introduce a wayefunction which provides a continuous transition from a localized to a delocalized form. Conventional band theory techniques provided such a function. Specifically, the single particle orbitals in the Slater determinant part of $\Psi_{T}$ were taken from an augmented plane wave (APW) calculation. The electron-nuclear Jastrow factor was suppressed as this cusp condition is correctly accounted for in the APW functions.

The APW method uses Bloch's theorem to reduce the description of a crystalline solid to a calculation in the primitive cell. This results in a set of coupled one particle Schrödinger equations which are solved by a discretization in reciprocal space. This discretization is introduced by considering only those functions which are periodic on the scale of a few unit cells.

The APW method builds solutions of the Schrödinger equation by solving the radial equation inside the mufin tin sphere and matcbing them to linear combinations of plane waves in the interstitial region. The APW wavefunctions are generated according to the formulae:

$$
\psi_{\boldsymbol{r}}\left(\vec{r}+\vec{F}_{j}\right)=\sum_{R} \sum_{i} c_{i} \Gamma_{11}(\mathbf{R}) e^{\vec{r} \cdot \mathrm{X}} \phi_{R \cdot \vec{r}}(\vec{r}) .
$$

where $c_{i}$ are the standard APW ccefficients $[15]$, a are the set of rotations leaving the crystal invariant, $\Gamma_{13}(r)$ are the matrix elements of the invariant group of the vector $k, \vec{k}_{i}=\vec{k}+\vec{K}_{i}\left(\vec{K}_{i}\right.$ a reciprocal lattice vector) and $\vec{X}_{j}$ is a lattice site. The function

$$
\phi_{\mathrm{R}} \overrightarrow{\vec{r}_{1}}(\vec{r})=\sum_{i, m} a_{i m}\left(R \overrightarrow{k_{i}}\right) u_{l}(r) Y_{l m}(\hat{r})
$$

is the muffin tin solution expressed as an expansion in spherical harmanics. Outside the muffin tin the solution is expressed as a sum of plane waves

$$
\nu_{T}(\vec{r})=\sum_{R} \sum_{i} c_{i} \Gamma_{21}(R) e^{i R T_{1} \cdot \vec{r}},
$$

where the $c_{i}$ are the matching constants from the APW functions of equation ( 8 ).

The APW calculations were performed using the same number of points ( $\vec{k}$ values) in the Brillouin zone as there were lattice sites in the quantum Monte Carlo calculation (32 for FCC, 16 for BCC). This provided the cortect number of single particle orbitals for the quantum Monte Carlo trial function. The maximum angulat momentum value 1 was taken to be 13 in the APW calculation and restricted to 3 in the subsequent generation of the wavefunction. The 1 values in the wavefunction could be limited to the $s, p, d$, and $f$ states since this was sufficient to represent the charge density. The APW calculations were performed for a few densities using an increased number of $l$ and $\vec{R}$ values without 
significant changes in the energy and pressure. The APW resuits agreed with the detailed band structure at normal and 10-5old compression [4] and with the energies and pressures [3] obtained from more accurate band theory calculations, to within the uncertainty caused by number dependence and exchange potential parameters. In particular, the APW results showed that the $2 \mathrm{~s}-2 \mathrm{p}$ band is not free-electron-like at 10-fold compression, Indicating that a plane wave trial function is a poor choice. At higher compressions, both the is and $2 s-2 p$ bands are free-electron-like and energies and pressures approximate Thomas-Fermi model results.

Table 1. Quantum Wonte Carlo variational and fxed-node diffusion energies for lithium at various densities. Energies are in Rydbergs. Densities are given in terms of the Wigner-Seitz radius r, and the compression. The calculations are for 16 and 32 atoms in the simulation box. The trial functions are Gaussian and plane wave ( $g-p w)$, all plane wave (pw) and APW (apw). Errors in the last digit are given in parentheses.

\begin{tabular}{|c|c|c|c|c|c|c|c|}
\hline \multicolumn{2}{|c|}{ Density } & \multicolumn{3}{|c|}{$\mathrm{M}=16$ atoms } & \multicolumn{3}{|c|}{$\mathrm{M}=32$ atoms } \\
\hline & Comn & tif & $E$ & $E_{t}$ & tf & $E_{\text {. }}$ & $E_{t}$ \\
\hline 3.500 & 0.263 & $g-p w$ & $-14.38(1)$ & $-14.87(1)$ & & & \\
\hline 3.000 & 0.418 & $g-P w$ & $-14.84(1)$ & $-15.01(1)$ & & & \\
\hline 2.500 & 0.722 & $g$-pw & $-14.90(1)$ & $-15.03(1)$ & & & \\
\hline 2.260 & 0.978 & $g-p w$ & $-14.91(1)$ & $-15.03(1)$ & $\begin{array}{r}\text { g-pw } \\
\text { apw }\end{array}$ & $\begin{array}{l}-14.82(1) \\
-14.87(1)\end{array}$ & $\begin{array}{l}-15.00(1) \\
-15.02(1)\end{array}$ \\
\hline 2.100 & 1.218 & g-pw & $-14.80(1)$ & $-15.03(1)$ & & & \\
\hline 2.000 & 1.412 & g-pw & $-14.88(1)$ & $-15.00(1)$ & apw & $-14.87(1)$ & \\
\hline 1.800 & 1.936 & g-pw & $-14.85(1)$ & $-14.96(1)$ & apw & $-14.84(1)$ & \\
\hline 1.600 & 2.757 & g-pw & $-14.74(1)$ & $-14.88(1)$ & apw & $-14.77(1)$ & \\
\hline 1.400 & 4.115 & & & & apw & $-14.66(2)$ & \\
\hline 1.250 & 5.783 & $g-p w$ & $-14.00(1)$ & $-14.3 \theta(1)$ & apw & $-14.49(3)$ & \\
\hline 1.000 & 11.289 & $g-p w$ & $-13.34(1)$ & $-13.43(1)$ & $g-p w$ & $-13.60(1)$ & $-13.78(1)$ \\
\hline & & pw & $-11.36(1)$ & $-12.57(2)$ & apw & $-13.89(1)$ & \\
\hline 0.800 & 22.087 & g-pw & $-11.22(1)$ & $-11.32(1)$ & g-pw & $-11.81(1)$ & $-11.93(1)$ \\
\hline & & $\mathrm{pw}$ & $-10.82(1)$ & $-11.77(1)$ & apw & $-12.89(1)$ & \\
\hline 0.750 & 26.766 & $\mathrm{~g}-\mathrm{pw}$ & $-10.30(1)$ & $-10.38(1)$ & & & \\
\hline & & pw & $-10.52(1)$ & $-11.33(1)$ & & & \\
\hline 0.700 & 32.840 & & & & apw & $-11.26(1)$ & \\
\hline 0.650 & 41.115 & g-pw & $-7.39(1)$ & $-7.49(1)$ & & & \\
\hline & & pw & $-9.45(1)$ & $-10.13(1)$ & & & \\
\hline 0.600 & 52.307 & & & & apw & $-0.46(1)$ & \\
\hline 0.500 & 90.334 & $\mathrm{pv}$ & $-5.13(2)$ & $-5.64(1)$ & pw & $-3.17(1)$ & \\
\hline 0.226 & 978.220 & $\mathrm{pw}$ & $61.70(4)$ & $61.50(1)$ & apw & $-5.12(1)$ & \\
\hline
\end{tabular}


Quantum Monte Carlo calculations were performed on a supercell with periodic boundary conditions, the atoms being located at fixed crystal lattice sites. Although fxed atomic sites are not required by the quantum Monte Carlo algorithm, in contrast to band theory methods, in practice regular BCC or FCC structures were simulated. Present calculations are not sufficiently accurate to determine the type of crystal structure. Different structures were used primarily to determine the number dependence correction. Simulations were performed on cubic supercells consisting of 48 or 182 electrons (16 or 54 atoms) for the BCC structure and 96 electrons ( 32 atoms) for the FCC structure. Experimental data as well as band theory calculations indicate a close packing structure (HCP or $\mathrm{FCC}$ ) for the $0^{\circ} \mathrm{K}$ isotherm at low pressures $[4][18 ![17\rfloor[18\rfloor$.

Preliminary calculations of lithin using 16 atoms per simulation box and simple trial Cunctions were performed over a compression range from 0.263 to 1000. Mixed Gaussian and plane wave trial functions were used at low to intermediate densities and planes waves alone at extreme compressions. The results are show in Table 1 and Figure 1. The large energy gap between the variational and diffusion energies for the plane vave trial function at intermediate compressions indicates that the trial function is not of an optimal form. It is necessary to reintroduce the electron-nuclear Jastrow factor and multiply both Jastrow terms

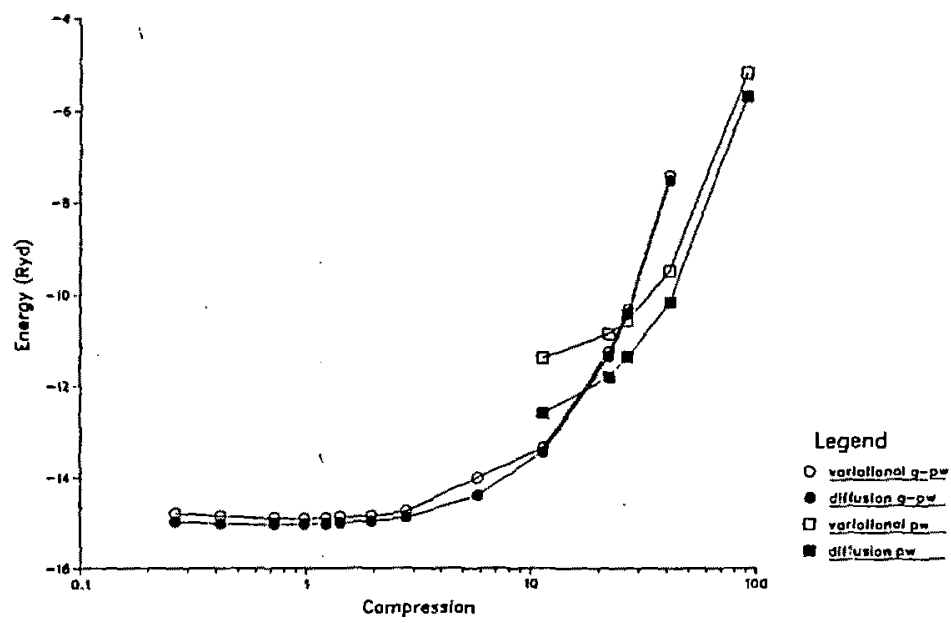

Figure 1. Variational and diffusion energies vs. Compression for quantum Monte Carlo calculations of lithium using 16 atoms in the simulation box. Trial functions are Gaussian is, plane wave 2s-2p ( $g-p w$ ) and plane wave 1s, $2 \mathrm{~s}-2 \mathrm{p}$ (pw) as indicated. Error bars are too small to be seen on this scale. 
by a variationally optimized parameter in order to incorporate more of the pair correlation energies. This should reduce the difference in the variational and difiusion energies as well as the discontinuity in the energies of the two types of trial functions at the crossing of the energy curves. This crossing near 25-fold compression indicates the approximate location of the delocalization of the $1 \mathrm{~s}$ core states. The result is consistent with band theory estimates of delocalization in the 30-300Mbar range.

Figure 2 shows the energies near normal density. The minimum of the curve gives an estimate for the equilibrium lattice constant which is consistent with the sccepted value of 6.48 a.u. For low to intermediate densities, the difference between the variational and diffusion energies is on the order of 0.1Rydbergs (see Table 1). Previous quantum Monte Carlo simulations of the electron gas at comparable densities yielded a lowering of the energy of 0.004 Rydbergs [10], indicating that the electron gas is a much simpler system than lithium. However, calculations of $\mathrm{Li}_{2}$ sho'ed a variational-difusion energy difference of about $0.04 \mathrm{Ryd}$ bergs [12]. Thus the trial functions rsed here for metallie lithium are of comparable quality to those used in the molecular case. The number dependence correction for metallic lithium is of the same order of magnitude as the variationaldiffusion energy difference (compare the results for 16 and 32 atoms in Table 1). At normal compression, the size correction of .1Rydbergs is the same as for the electron gas of the same density.

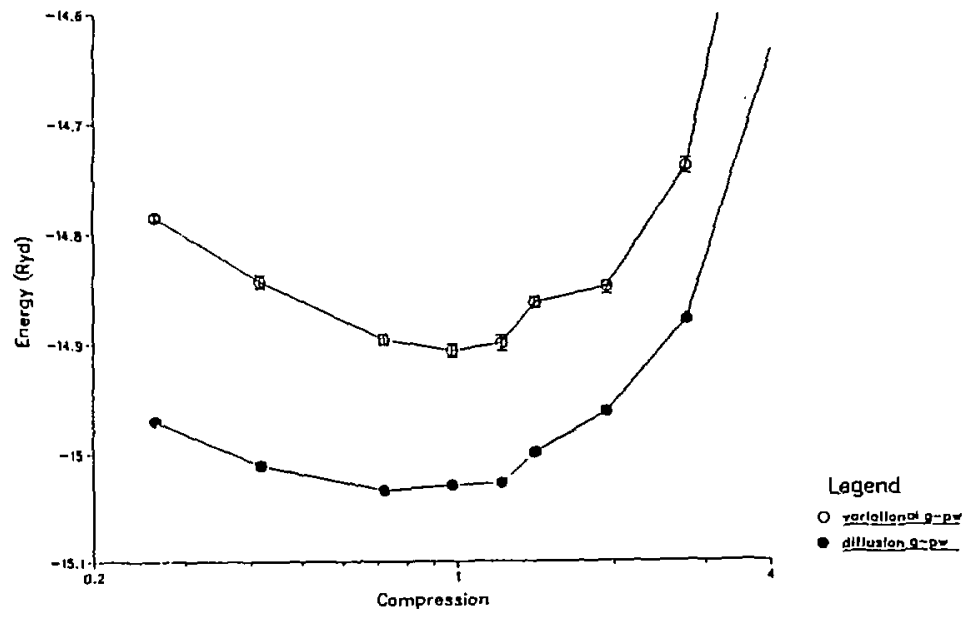

Figure 2. Variational and diffusion energies vs. Compression for quantum Monte Carlo calculations of lithium using 16 atoms in the simulation box near normal density and Gaussian $1 \mathrm{~s}$, plane wave 2s-2p ( $g-p w$ ) trial Iunctions. 
The calculations bave been carried out for crystals expanded to 4 times the normal density using trial functions with Gaussian 1s and plane wave $2 s$ orbitals. Additional siruulations are underway using localized states (Hermann-Skillman, simple Gaussian, or Wannier) for all the electrons. The density at which the energy curves for the two kinds of trial functions cross will then locate the Mott transition - the delocalization of the $2 \mathrm{~s}$ states.

In order to study the core delocalization regime and for comparison with band theory, a 32 particle crystal was simulated using both simple and APW trial functions. The APW trial function was a factor of $t-5$ times slower to sample than the simple Gaussian-plane wave form. This was offset by the significant lowering of the variational energies (see the preliminary results shown in Figure 3 and Table 1) and should also be reflected by a more rapid convergence in the diffusion calculations. To compare with band theory, the energies relative to that at normal density are plotted in Figure 4. The variational quantum Monte Carlo values are in reasonable agreement with both the ASW [3] and the LMTO [2] results. At extreme compressions, the variational values satisfy the $P V^{5 / 3}$ equation of state of Thomas-Fermi-Dirac models.

More calculations are required before the quantum Monte Carlo algorithm can be used to assess the accuracy of band theories. The Jastrow terms for both the simple and the APW trial functions must be optimized and used in iiffusion and Green's function runs at selected densities. These simulations are presently underway. Dependence of the results on the number of particleg must be taken into account by extrapolation to the infinite particle number limit using a fit for several supercell sizes at soveral densities [10]. The number dependence may not

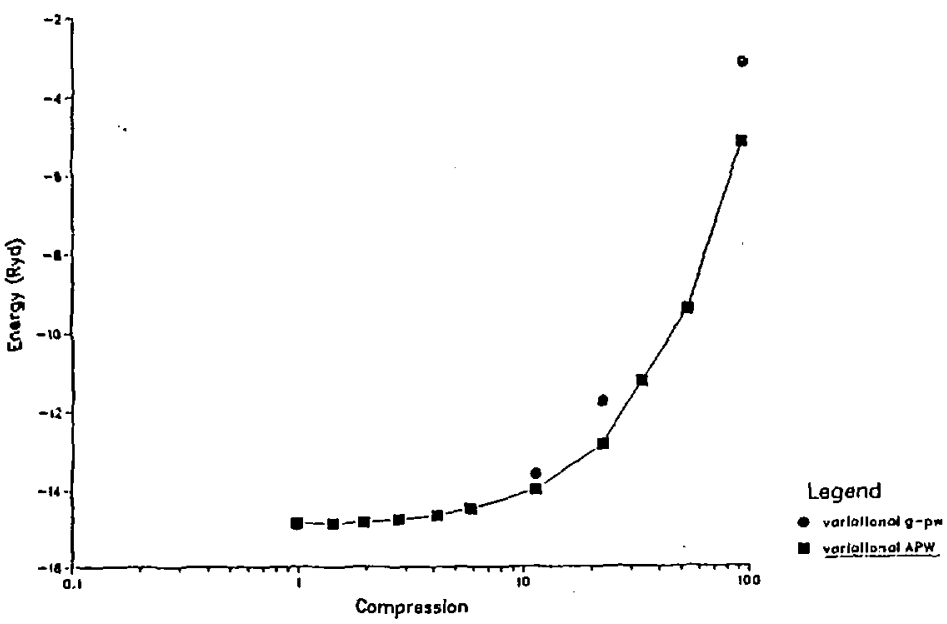

Figure 3. Preliminary variational energies vs. Compression for quantum Monte Carlo calculations of lithium using 32 atums in the simulation box and Gaussian- plane wave ( $\mathrm{g}-\mathrm{pw}$ ) or APW trial functions. 


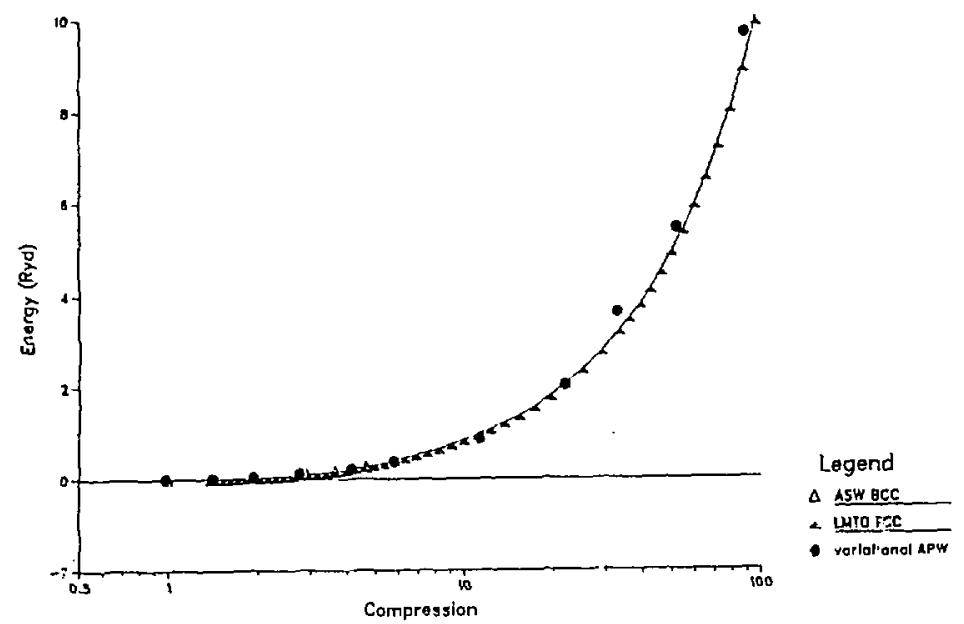

Figure 4. Relative Energy vs. Compression for band theory ASW [3] and LMTO [2] calcuiations and variational quantum Monte Carlo calculations using APW' trial functions.

be the same for different trial function forms or for different numbers of delocalizated electrons. Particular emphasis will be given to the delocalization regimes of the $2 s-2 p$ electrons at low density (Mott transition) and the $1 \mathrm{~s}$ electrons at high density - regions where the largest correlation effects should occur.

\section{ACKNOWLEDGEMENCS}

We wish to express our appreciation to M.Bukovinski for supplying the APW codes, to A. McMahan for his advice on band theory and especially to D.Ceperley for sharing his expertise on quantum Monte Carlo. We also wish to thank the San Diego Supercomputing Center for providing computer time.

This work was performed under the auspices of the Department of Energy Lawrence Livermore National Laboratory Contract No. W-7405-ENG-48. 


\section{REFERENCES}

[1] D.Liberman, Colloques Internationaux de la Centre de la Recherche Scientifique No. 188: 35 (1969)

(2) A.MrMahan, private communication

(3) W.Zittel, J.Meyer-ter-Vehn, J.Boettger and S.Trickey, J. Phys. F15: L247 (1885)

[4] J.Boettger and S.Trickey, Phys, Rev. B32: 3391 (1985)

[5] A.Bakanova, I.Dudoladov, R.Trunin, Soviet Physicz - Solid State 7: 1307 (1985)

[8] R.Grover, R.Keeler, F.Roger, G.Kennnedy, J. Phys. Chem. Solids 30: 2091 (1969)

[7] R.M.More, Phys. Rev. A19: 1234 (1970)

[8] F.Perrot, Physica 98A: 555 (1879)

[9] D.Ceperley and B.J.Adder, Science 231: 550 (1986)

(10] D.Ceperley and B.J.Alder, Phys. Rev, Lett. 45: 566 (1980)

[11] D.Ceperley, Phys. Rev, B18: 3126 (1978)

[12] P.Reynolds, D.Ceperley, B.J.Alder, W.Lester, J. Chem. Phys. 77: 5593 (1982)

[13] D.Ceperley and B.Alder, J. Chem. Phys. 81: 5833 (1984)

[14] c.f. Reference [9] and D.Ceperley and B.Alder, publication in preparation

[15] L.Mattheis, J.Wood, A.Switendick, Methods in Comp. Physics, Vol. 8: 63 (1968)

[16] H.Skriver, Phys. Rev. B31: 1909 (1985)

[17] D.Young and M.Ross, Phys. Rev. B29: 682 (1084)

[18] T.Lin and K.Dunn, Phys. Rev. B33: 807 (1086) 